\title{
Rare giant primary ureteral polyp: A case report and literature review
}

\author{
YUNLIN CAI, ZONGPING ZHANG and XIAOFENG YUE \\ Department of Urology, The Second Clinical Hospital of North Sichuan Medical College \\ (Nanchong Central Hospital), Nanchong, Sichuan 637000, P.R. China
}

Received June 10, 2016; Accepted January 31, 2017

DOI: $10.3892 / \mathrm{mco} .2017 .1146$

\begin{abstract}
Primary ureteral fibroepithelial polyps (UFPs) are rare benign tumors of mesodermal origin. The majority UFPs are observed in the proximal part of the ureter and most commonly on the left side. The mean diameter of UFP is reported to be $<5 \mathrm{~cm}$. We herein present a rare case of a long primary ureteral polyp originating from the lower ureter in a 53-year-old woman. Following computed tomography, magnetic resonance imaging, retrograde pyelography and ureteroscopic examination, a preliminary diagnosis of giant primary lower ureteral polyp protruding into the bladder was obtained. Polyp resection and resection of the narrow ureteral segment with a V-shaped end-to-end anastomosis were performed. The length of this polyp was $15.0 \mathrm{~cm}$, and the final pathological diagnosis was UFP. No recurrence or ureteral stenosis were observed at the 12-month follow-up. A supplementary review of previously published cases and related literature is also included.
\end{abstract}

\section{Introduction}

Primary ureteral polyps are rare benign tumors, comprising $<1 \%$ of all genitourinary neoplasms (1). The presenting symptoms are vague; thus, preoperative diagnosis may be difficult. According to a recent systematic literature review, the median size of these polyps is $4.0 \mathrm{~cm}$ (2). However, polyps $>15 \mathrm{~cm}$ have also been reported, although they are usually found in the proximal ureter. Due to its rarity, there is no standard treatment for this disease; however, complete excision is the optimal method to avoid recurrence. We herein present the case of a patient with a giant primary ureteral polyp located in the lower part of the ureter in an older woman, and discuss the diagnostic and therapeutic measures and related literature.

Correspondence to: Professor Zongping Zhang, Department of Urology, The Second Clinical Hospital of North Sichuan Medical College (Nanchong Central Hospital), Renmin South Road, Nanchong, Sichuan 637000, P.R. China

E-mail: 419993729@qq.com

Key words: giant primary ureteral polyps, ureteroscopy, lower ureter

\section{Case report}

A 53-year-old woman was admitted to the Second Clinical Hospital of North Sichuan Medical College (Nanchong, China) with gross painless hematuria for $\sim 2$ years and frequency of urination for $\sim 1$ month. The physical examination was unremarkable. Multidetector computed tomography urography (CTU) revealed a filling defect in the lower part of the right ureter (Fig. 1). Cystoscopy revealed a mass $\sim 3 \mathrm{~cm}$ in length, with a smooth surface, protruding from the ureteral orifice into the bladder (Fig. 2). Examination of a bioptic specimen suggested that the mass was an inflammatory polyp. A $5 \mathrm{~F}$ ureteral catheter was inserted from the right ureteral orifice; $\sim 10 \mathrm{~cm}$ after the insertion point, resistance was encountered and the catheter could not be advanced further. Retrograde urography was subsequently performed and the contrast media failed to reach the upper part of the ureter and the renal pelvis, whereas the lower part of the ureter exhibited a filling defect (Fig. 3).

Ureteroscopic assessment was performed under general anaesthesia and revealed a pedunculated tumor $(\sim 15 \mathrm{~cm}$ in length) arising from the lower segment of the right ureter, with a smooth surface, with the base of the tumor located $\sim 13 \mathrm{~cm}$ from the ureteral orifice. The tumor macroscopically resembled a long grape. As we unable to find the upper ureteral orifice, the ureteroscope and guidewire could not pass through the base of the tumor. A small piece of tumor was resected by Holmium laser for histopathological examination and a Double-J stent was inserted, with the upper end of the stent located in the base of the tumor. Resectoscopy was then performed to incise the portion of the polyp protruding into the bladder. The histological examination of these two bioptic specimens suggested that the tumor was a fibroepithelial polyp. Kidney-ureter-bladder imaging was then performed, which revealed that the Double-J stent was folded and twisted in the fifth lumbar plane (Fig. 4).

Open surgery was employed under general anaesthesia, Resection of the polyp and the narrow ureteral segment plus $\mathrm{V}$-shaped end-to-end ureteral anastomosis were performed. An oblique incision on the right low abdomen was performed. Following exposure of the ureter, taking the apex of the Double J-tube as the longitudinal excision sign, the ureter was longitudinally opened for $\sim 2 \mathrm{~cm}$, the root of the polyp was immediately identified, and the ureter above the polyp was 


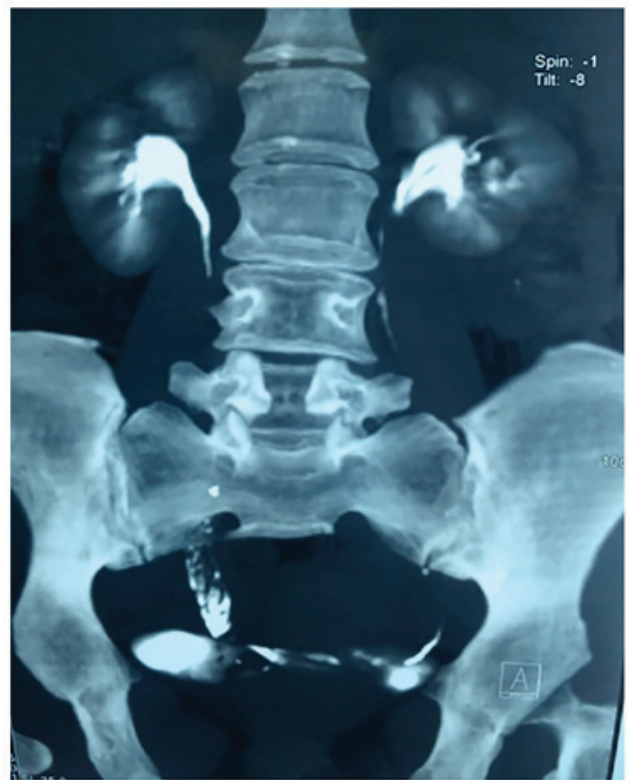

Figure 1. Computed tomography urography showing a filling defect at the lower part of the right ureter.

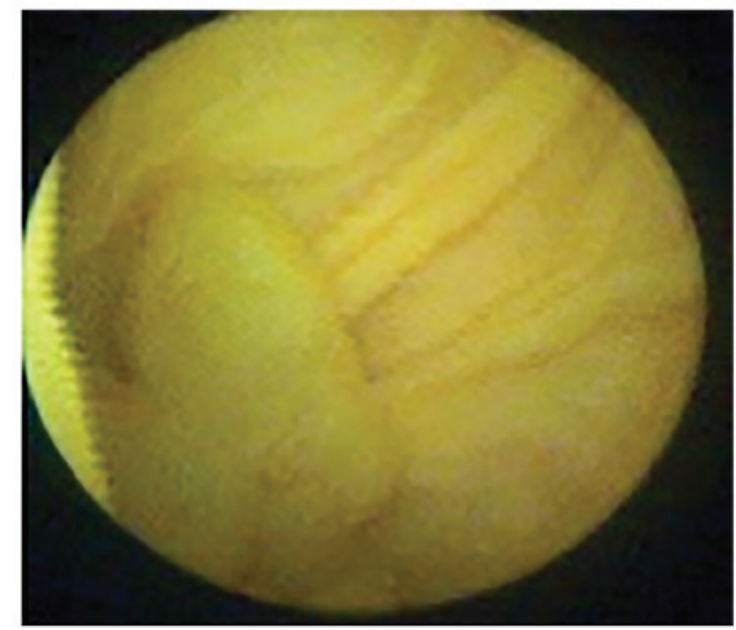

Figure 2. Cystoscopy showing a neoplasm with a smooth surface, $\sim 3 \mathrm{~cm}$ in length, arising from the right ureter.

narrowed. The polyp was cut at the base and was completely removed from the ureter (Fig. 5); the length of the polyp was $\sim 12 \mathrm{~cm}$ (Fig. 6). Approximately $0.5 \mathrm{~cm}$ of the narrow ureteral segment was resected and end-to-end anastomosis was performed. A Double-J stent was left in place.

The postoperative histopathological examination findings revealed that the tumor was a ureteral fibroepithelial polyp (UFP). The Double-J stent was removed 2 months after the operation. The patient was asymptomatic, and no complications or recurrence have occurred during the 12 months of follow-up. gave their informed consent regarding the publication of the case details.

\section{Discussion}

Primary ureteral tumors are among the rarest in the spectrum of genitourinary tumors and are most commonly malignant.

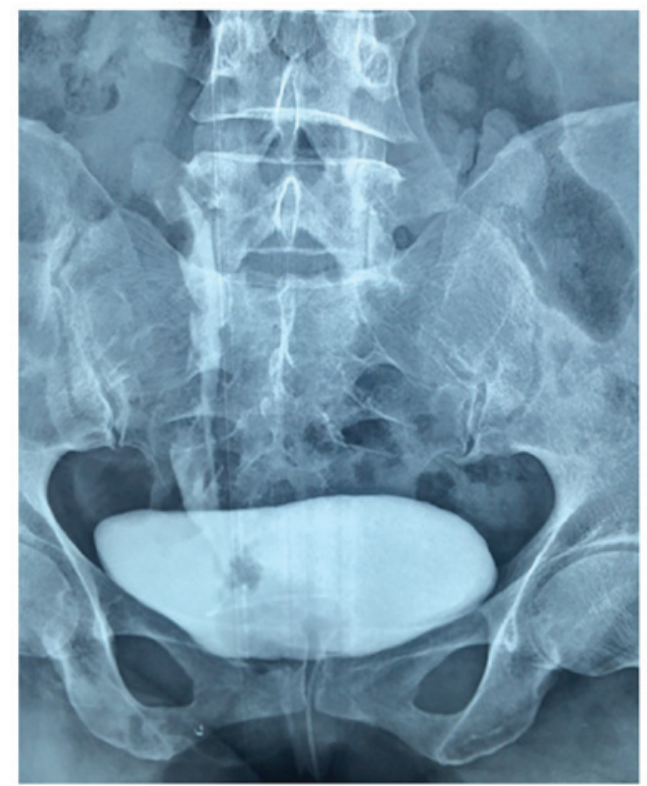

Figure 3. Retrograde urography showing a filling defect in the right lower ureter and no contrast medium in the upper ureter and renal pelvis.

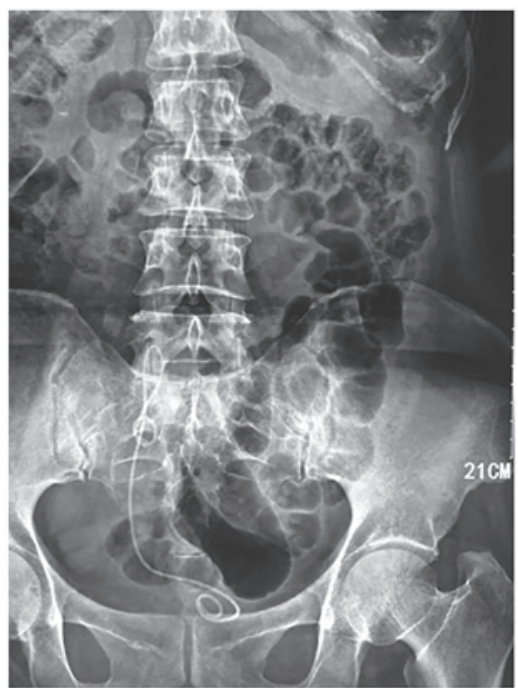

Figure 4. Kidney-ureter-bladder ultrasound, showing the Double-J stent folded and twisted in the fifth lumbar plane.

Only one-fifth of these tumors are benign and, among those, UFPs are considered to be the most common. The etiology of benign ureteral polyps is unclear. They are considered to be the result of various factors, including congenital (developmental anomaly), obstruction, trauma, irritation, infection, and specific endogenous hormonal imbalances (3). The clinical presentation of primary UFPs is non-specific. They most commonly present as a single, small polyp. Reports of multiple, bilateral polyps are extremely rare, whereas the mean diameter of UFPs was reported to be $<5 \mathrm{~cm}$; larger polyps may extend into the bladder cavity and may be difficult to distinguish from bladder tumors $(4,5)$. In the majority of the cases, the upper ureter is the most common site of origin of these tumors, whereas polyps derived from the lower urinary tract are not as frequent (6). UFPs most commonly occur between 


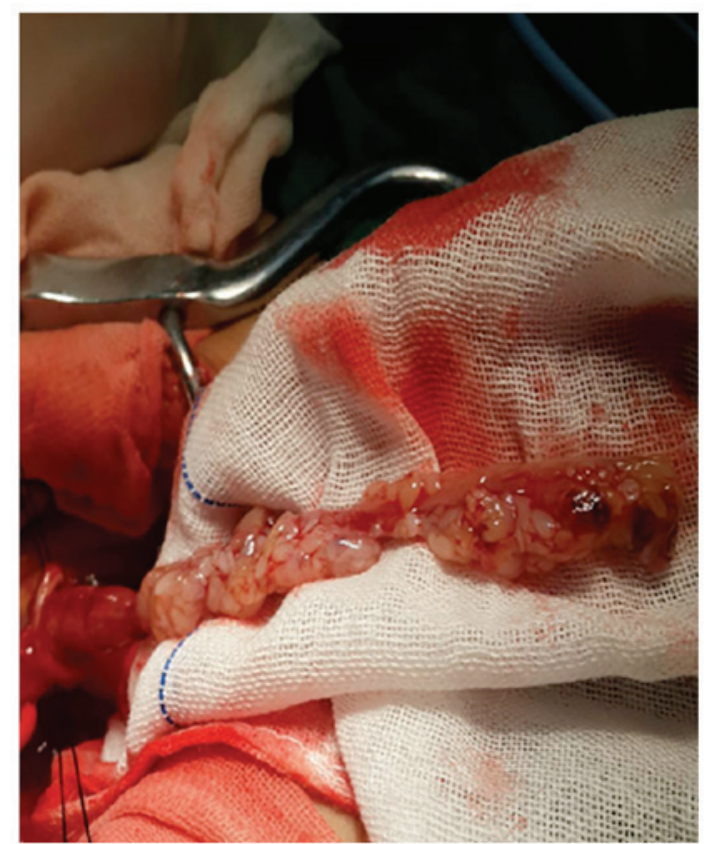

Figure 5. Gross appearance of the excised ureteral polyp, with a smooth surface and shaped similar to a long grape brunch.

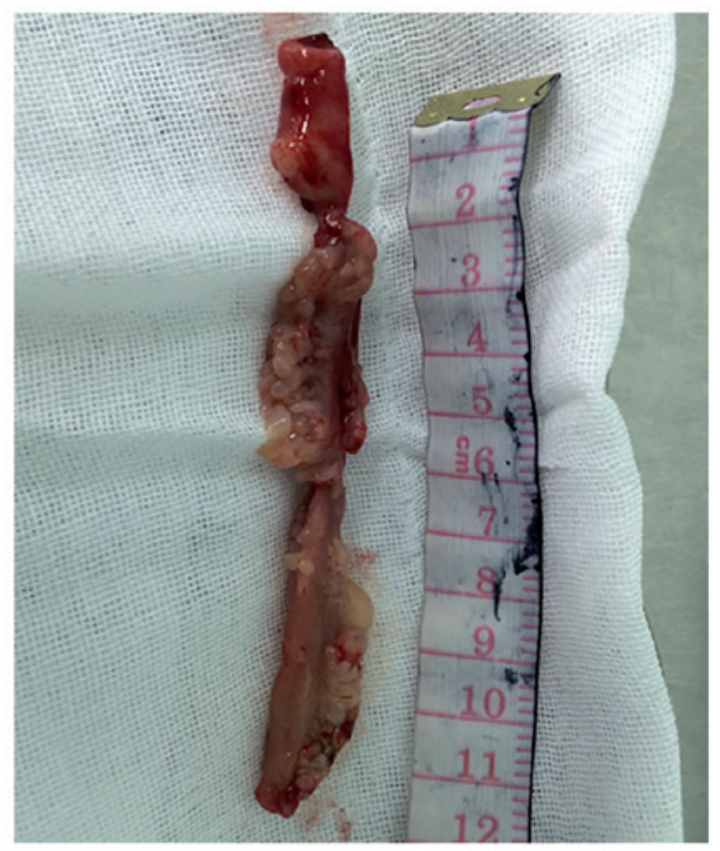

Figure 6 . The $12.0-\mathrm{cm}$ long fibroepithelial polyp removed by open surgery from the right ureter.

the second and fourth decades, and they usually originate from the left ureter. The clinical symptoms are non-specific and the majority of the early primary UFPs are asymptomatic. Necrosis and bleeding on the polyp surface may be expressed as gross hematuria and, when obstruction occurs, hydronephrosis or renal colic may develop. In the present case, the polyp occurred in the lower part of the right ureter in a middle-aged woman; in addition, it was broad-based and had a total length of $\sim 15 \mathrm{~cm}$ (portion removed by open surgery, $\sim 12 \mathrm{~cm}$; and ureteroscopically removed bladder part, $\sim 3 \mathrm{~cm}$ ).
Previous reports of such lower-segment ureteral giant primary UFPs are extremely rare.

Imaging examination (7) may be helpful and suggestive of a UFP diagnosis. Intravenous pyelography or CTU may show a filling defect, thereby hinting at the state of the renal function of the patient, and may also reveal whether hydronephrosis may be present. However, preoperative radiographic diagnosis may be challenging, as UFPs usually present as a filling defect, which may be attributed to blood clots, radiolucent calculi, neoplasms or a crossing vessel. Moreover, it may be difficult to differentiate UFPs from transitional cell carcinoma based only on imaging findings (8), as preoperative diagnosis confirmation is difficult and $\mathrm{Li}$ et al reported in 2014 that none of the UFPs in their study were detected prior to surgery (9). Mistaking these tumors for transitional cell carcinomas may result in unnecessary nephroureterectomy.

For patients with suspected UFPs who have been evaluated by intravenous pyelography, CT, or retrograde urography, a ureteroscopic examination is also required to confirm the diagnosis. Ureteroscopy may be attempted to obtain a diagnosis and determine the optimal treatment approach. Prior to ureteroscopy becoming widespread, differentiating malignant from benign lesions was difficult. The number of polyps, the diameter of the base and the location of the obstruction may be determined through ureteroscopy in the same session; ureteroscopic biopsy of the lesion may be performed for larger tumors, whereas smaller tumors may be completely resected during ureteroscopy, thus avoiding a second surgery. In the present case, preoperative ureteroscopic examination revealed that the polyp was extremely long, originated from the lower part of the ureter and its base was wide; the biopsy result revealed a benign fibroepithelial polyp, and surgical resection was selected. At the same time, a Double-J stent was also placed at the obstruction site, which was identified by searching the base of the polyp during open surgery.

The management of UFPs currently depends on the site, size and clinical expertise. Smaller lesions may simply be fulgurated endoscopically, while larger lesions require proper surgical excision. With the advent of new technology, minimally invasive techniques have become popular. Previous reports have described successful polypectomy through the use of ureteroscopy and endoscopic treatment $(5,10)$. However, ureteroscopy and endoscopic resection may be difficult in patients with long or large polypoid lesions, due to poor visualization of the base of the stalk and limited working space, which make it difficult to differentiate the ureteral wall from the polyp, leading to incomplete resection or ureteral perforation. Laparoscopic surgery is another minimally invasive method $(11,12)$. Successful laparoscopic treatment has been described in patients with large, long polyps, as well as in those with multiple polyps. Kijvikai et al (13) described the transperitoneal laparoscopic management of a $17-\mathrm{cm}$ long fibroepithelial polyp in the proximal ureter associated with ureteral obstruction. However, this approach may not be suitable for polyps in the lower part of the ureter, particularly in cases without hydronephrosis, and laparoscopic surgery may cause difficulties in ureteral anastomosis following ureteral resection, particularly in patients with a lower ureteral polyp. Complete resection is considered the optimal method for avoiding recurrence of UFPs, as incomplete resection may 
result in recurrence. In our case, as the polyp was long, its stalk could not be fully visualized via ureterorenoscopy; furthermore, it occurred in an older woman and originated from the lower part of the ureter. Our main objective was to ensure complete resection, so open surgery was selected in this case. A small ureteral segment was resected, including the narrowed potion of the ureter and the entire stalk of the polyp, followed by end-to-end ureteral anastomosis.

In summary, an extremely long pedunculated UFP of the distal ureter, which protruded into the bladder, was excised via open surgery. Pedunculated urothelial polyps originating from the ureter should be taken into consideration in the differential diagnosis of a bladder mass on imaging. Primary ureteral polyps are benign and the prognosis is usually good. Ureteroscopy is not only a useful diagnostic method, but may also be an effective treatment for smaller, solitary ureteral polyps. For larger polyps, in patients with obstruction, ureteroscopy may help determine the polyp size, its location, the location of the ureteral stenosis, as well as the base of the polyp, which may help determine the optimal surgical approach.

\section{Acknowledgements}

The authors would like to thank Anguo Wang for assisting with the surgical procedure. This study was supported by the North Sichuan Medical College (grant no. 20131252).

\section{References}

1. Piovesan AC, Torricelli FC, Borges LL, Chambô JL, Mesquita JL and Srougi M: Ureteral fibroepithelial polyps in a pregnant woman: Case report. Sao Paulo Med J 127: 238-240, 2009.
2. Ludwig DJ, Buddingh KT, Kums JJM, Kropman RF, Roshani H and Hirdes WH: Treatment and outcome of fibroepithelial ureteral polyps: A systematic literature review. Can Urol Assoc J 9: E631-E637, 2015.

3. Ye L, Zhao LJ, Yue F, Song XS, Wei W, Jiang XJ and Yang JY: Large ureteral fibroepithelial polyp lacking epithelium due to ischemic infarction. Kaohsiung J Med Sci 28: 457-461, 2012.

4. Kaba M, Kaba S, Kaya TY, Eren H, Pirincci N: A giant pedunculated urothelial polyp imicking bladder mass in a child: a rare case. Case Rep Pediatr 2014, 2014:1 3.

5. Liu C, Liu XJ, Liu D and Yao DW: A giant ureteral polyp mimicking as a bladder mass resected ureteroscopically by diode laser: A case report and literature review. Int J Clin Exp Pathol 8: 14580-14583, 2015.

6. Childs MA, Umbreit EC, Krambeck AE, Sebo TJ, Patterson DE and Gettman MT: Fibroepithelial polyps of the ureter: A singleinstitutional experience. J Endourol 23: 1415-1419, 2009.

7. Xu C, Zhang Z, Ye H, Wu C, Zhang C, Zhang Y, Wang Y, Cao Z, Wang $\mathrm{H}, \mathrm{Xu} \mathrm{W}$, et al: Imaging diagnosis and endoscopic treatment for ureteral fibroepithelial polyp prolapsing into the bladder. J Xray Sci Technol 21: 393-399, 2013.

8. Patheyar V, Venkatesh SK, Siew EP, Consiglieri DT and Putti T: MR imaging features of fibroepithelial ureteral polyp in a patient with duplicated upper urinary tract. Singapore Med J 52: e45-e 47, 2011.

9. Li R, Lightfoot M, Alsyouf M, Nicolay L, Baldwin DD and Chamberlin DA: Diagnosis and management of ureteral fibroepithelial polyps in children: A new treatment algorithm. J Pediatr Urol 11: 22.e1-22.e6, 2015.

10. Hubosky SG and Bagley DH: Laser Resection of Fibroepithelial Polyps with Digital Ureteroscopy. J Endourol Case Rep 1: 36-38, 2015.

11. Dai LN, Chen CD, Lin XK, Wang YB, Xia LG, Liu P, Chen XM and Li ZR: Retroperitoneal laparoscopy management for ureteral fibroepithelial polyps causing hydronephrosis in children: A report of five cases. J Pediatr Urol 11: 257.e1-257.e5, 2015.

12. Bian Z, Liu X, Hua Y, Liu F, Lin T and He D: Laparoscopic management of multiple ureteral polyps in children. J Urol 186: 1444-1449, 2011

13. Kijvikai K, Maynes LJ, Herrell SD: Laparoscopic management of large ureteral fibroepithelial polyp. Urology 70: 373, 2007. 